\title{
The Size and Shape of a Single Photon
}

\author{
Zhenglong Xu \\ Wuzhen High School, Tongxiang, China \\ Email: zhl-xu@126.com
}

How to cite this paper: Xu, Z.L. (2021) The Size and Shape of a Single Photon. Open Access Library Journal, 8: e7179. https://doi.org/10.4236/oalib.1107179

Received: January 24, 2021

Accepted: February 23, 2021

Published: February 26, 2021

Copyright $\odot 2021$ by author(s) and Open Access Library Inc.

This work is licensed under the Creative Commons Attribution International License (CC BY 4.0).

http://creativecommons.org/licenses/by/4.0/

\section{(c) (i) Open Access}

\begin{abstract}
The size and shape of a single photon is a property of photon shown in the collision of the photon with charged particles. Applying scattering theory and classical electrodynamics is the most reliable way to solve this problem. The main intention of this paper is to reveal the electromagnetic structure of a single photon. It establishes the formulas for the Poynting vector (energy flow vector) and the energy density. Applying the new explanation of Compton wavelength creates the probability formula for the Compton effect when a photon collides with an electron. The research discovers that the electromagnetic field of a single linearly polarized photon confines to a membrane-like cuboid, while the electromagnetic field of a single circularly polarized photon confines to a membrane-like shell of a hollow cylinder.
\end{abstract}

\section{Subject Areas}

Modern Physics

\section{Keywords}

Wave-Train Length, Cross-Sectional Area, Electromagnetic Field Structure, Poynting Vector, Polarized Photon

\section{Introduction}

In electrodynamics, the Maxwell equations and their solutions characterize the electromagnetic field of light. In quantum mechanics, the Planck energy formula represents the energy of a single photon. So far, for a single photon, the known physical quantities include velocity $c$ in the vacuum, the frequency $v$ or angular frequency $\omega=2 \pi v$, the wavelength $\lambda=c / v=2 \pi c / \omega$, the energy $\mathcal{E}=h v=\hbar \omega$, the momentum $p=h v / c=\hbar \omega / c$, etc. The connection of the light wave in electrodynamics and photon in quantum mechanics is not well. There are many mysteries about this issue since Max Planck introduced the concept of 
quantum, December 1900. For a single photon, it seems that there are still many physical quantities unknown. For example, does a single photon have size and shape? The textbooks are silent on this point. Most academic articles complicate this problem so much that it remains unsolved for 120 years. The photon models proposed by many scholars can't stand up to theoretical scrutiny. Therefore, revealing the size, shape, and internal electromagnetic structure of a single photon has become an indispensable part of photon research. Then, how to go deep into the interior of a single photon?

\section{Compton Scattering}

Applying the Thomson scattering and Compton scattering theory can reveal the cross-sectional area of the electron. One can't help asking whether Compton scattering theory can derive the cross-sectional area of a single photon.

Regarding the collision of the X-ray photon and the free electron as an elastic collision, both photon and electron are elastic particles (Figure 1). The particles obey the laws of conservation of momentum and kinetic energy.

According to the laws of conservation of momentum and kinetic energy can obtain the following equations [1]

$$
\left\{\begin{array}{l}
\beta m c^{2}+\hbar \omega^{\prime}=m c^{2}+\hbar \omega \\
p^{\prime} \cos \theta+p \cos \varphi=p_{0} \\
p^{\prime} \sin \theta-p \sin \varphi=0
\end{array}\right.
$$

The relevant physical quantities above and below are: $h$-Planck constant, $\hbar$-the reduced Planck constant, $\hbar=h / 2 \pi ; \hbar \omega$-the incident photon energy; $\hbar \omega^{\prime}$ - the scattered photon energy; $\boldsymbol{p}_{0}$-the incident photon momentum vector which magnitude is $p_{0}=\hbar \omega / c ; \boldsymbol{p}^{\prime}$-the scattered photon momentum vector which magnitude is $p^{\prime}=\hbar \omega^{\prime} / c ; m$-the mass of the electron; $V$-the speed of recoil electron; $\boldsymbol{p}$-the recoil electron momentum vector which magnitude is $p=\beta m V$ where $\beta=1 / \sqrt{1-V^{2} / c^{2}} ; \theta$-the scattering angle of the scattered photon; $\varphi$-the recoil angle of the scattering electron.

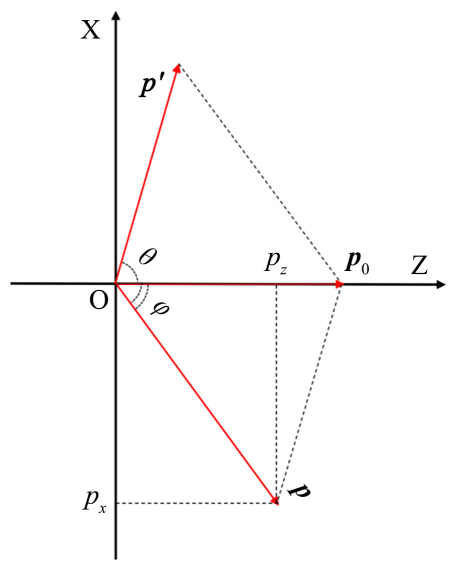

Figure 1. This diagram shows the conservation of momentum when a photon scattering by an electron at rest. 
The solutions to this system of equations are

$$
\left\{\begin{array}{l}
\lambda^{\prime}-\lambda=\lambda_{C}(1-\cos \theta) \\
\operatorname{ctg} \varphi=(1+\kappa) \operatorname{tg} \frac{\theta}{2} \\
p=\frac{2 \hbar \omega}{c} \frac{(1+\kappa) \cos \varphi}{1+2 \kappa+\kappa^{2} \sin ^{2} \varphi}
\end{array}\right.
$$

where $\lambda_{C}$ is Compton wavelength, which is given by

$$
\lambda_{C}=\frac{h}{m c}
$$

and $\kappa$ is the ratio of photon energy to the mass-energy of electron at rest, which is given by

$$
\kappa=\frac{\hbar \omega}{m c^{2}}
$$

In this set of solutions, Equation (2-1) represents the change in the wavelength of the photon before and after the collision; Equation (2-2) represents the relationship between the recoil angle of scattering electron and the scattering angle of the scattered photon; Equation (2-3) represents the recoil momentum of scattering electron.

The recoil momentum of the electron can decompose into two components: one in the $x$-axis perpendicular to the direction of the incident photon and another in the $z$-axis parallel to the direction of the incident photon

$$
\left\{\begin{array}{l}
p_{x}=-\frac{2 \hbar \omega}{c} \frac{(1+\kappa) \cos \varphi \sin \varphi}{1+2 \kappa+\kappa^{2} \sin ^{2} \varphi} \\
p_{z}=\frac{2 \hbar \omega}{c} \frac{(1+\kappa) \cos ^{2} \varphi}{1+2 \kappa+\kappa^{2} \sin ^{2} \varphi}
\end{array}\right.
$$

The energy gained by the recoil electron is equal to that lost by the photon. Applying Equation (2-1) can obtain the expression of the energy

$$
\Delta \mathcal{E}=\hbar \omega \frac{2 \kappa \cos ^{2} \varphi}{1+2 \kappa+\kappa^{2} \sin ^{2} \varphi}
$$

The domains of the angles are $0 \leq \theta \leq \pi, \quad 0 \leq \varphi \leq \pi / 2$.

\section{The Structure of Linearly Polarized Photon}

For the sake of simplicity, it is necessary to assume:

1) The photon energy is far less than the mass-energy of an electron at rest, i.e., $\hbar \omega \ll m c^{2}$.

2) The electron is a point-like particle of neither size nor shape.

3) The motion of the electron is undamped.

4) There is no effect of the photon magnetic field on the electron.

5) The motion of the electron is low-speed without the relativistic effect. 


\subsection{Wave Function and Poynting Vector}

A plane electromagnetic wave propagating along the $z$-axis, which electric field strength vector $E$ oscillating along the $X$-axis and the magnetic induction strength vector $\boldsymbol{B}$ oscillating along the $\mathrm{y}$-axis, obeys Maxwell equations. Both strengths can be characterized by the function as follows

$$
\left\{\begin{array}{l}
\boldsymbol{E}=E_{0} \sin \left[\omega\left(t-\frac{z}{c}\right)\right] \boldsymbol{e}_{x} \\
\boldsymbol{B}=B_{0} \sin \left[\omega\left(t-\frac{z}{c}\right)\right] \boldsymbol{e}_{y}
\end{array}\right.
$$

where $\boldsymbol{e}_{x}, \boldsymbol{e}_{y}$ are basis vectors.

It is undoubtedly correct to characterize a single photon by the same harmonic functions as light waves. However, the electromagnetic field strength of a single photon is different from that of light waves in two aspects. First, the photon function of the electromagnetic field strength is constrained to the photon energy (frequency or angular frequency), but that of the light wave is not. Second, the phase $\omega(t-z / c)$ or wave-train length of a single photon (photon length) is constrained to the photon energy (frequency or angular frequency), but that of the light wave is not.

The relationship between the electric field strength $E$ and the vector potential $A$ accords with the following formula

$$
\boldsymbol{E}=-\frac{\partial \boldsymbol{A}}{\partial t}
$$

By integrating $E$ over time t can obtain the expression of the vector potential $\boldsymbol{A}$

$$
\boldsymbol{A}=-\int \boldsymbol{E} \mathrm{d} t=\frac{E_{0}}{\omega} \cos \omega\left(t-\frac{z}{c}\right) \boldsymbol{e}_{x}
$$

The curl of $A$ can express as the following

$$
\begin{aligned}
\nabla \times \boldsymbol{A} & =\left(\frac{\partial}{\partial x} \boldsymbol{e}_{x}+\frac{\partial}{\partial y} \boldsymbol{e}_{y}+\frac{\partial}{\partial z} \boldsymbol{e}_{z}\right) \times\left[\frac{E_{0}}{\omega} \cos \omega\left(t-\frac{z}{c}\right) \boldsymbol{e}_{x}\right] \\
& =\frac{E_{0}}{c} \sin \omega\left(t-\frac{z}{c}\right) \boldsymbol{e}_{y}
\end{aligned}
$$

Employing the relationship between the amplitude of electric field strength and amplitude of magnetic induction strength, $B_{0}=E_{0} / c$, then compares with Equation (7-2), can conclude that

$$
\boldsymbol{B}=\nabla \times \boldsymbol{A}
$$

The spin angular momentum flow can express as

$$
\begin{aligned}
\boldsymbol{s} & =c \varepsilon_{0} \boldsymbol{E} \times \boldsymbol{A} \\
& =c \varepsilon_{0}\left[E_{0} \sin \omega\left(t-\frac{z}{c}\right) \boldsymbol{e}_{x}\right] \times\left[\frac{E_{0}}{c} \sin \left(t-\frac{z}{c}\right) \boldsymbol{e}_{x}\right]=0
\end{aligned}
$$

where $\boldsymbol{e}_{x} \times \boldsymbol{e}_{x}=0$. 
The result shows that the spin angular momentum flow is equal to zero. Therefore, there is no spin phenomenon in linearly polarized photons.

The energy flow vector of the electromagnetic field called "Poynting vector" [2] is given by

$$
\begin{gathered}
\mathcal{P}=\frac{\boldsymbol{E} \times \boldsymbol{B}}{\mu_{0}}=\frac{E_{0} \times B_{0}}{\mu_{0}} \sin ^{2} \omega\left(t-\frac{z}{c}\right) \boldsymbol{e}_{x} \times \boldsymbol{e}_{y} \\
=\mathcal{P}_{0} \sin ^{2} \omega\left(t-\frac{z}{c}\right) \boldsymbol{e}_{z}
\end{gathered}
$$

The above function observed at $z=0$ can be represented by

$$
\mathcal{P}=\mathcal{P}_{0} \sin ^{2} \omega t \boldsymbol{e}_{z}
$$

where the amplitude value of the Poynting vector in the formula is given by

$$
\mathcal{P}_{0}=\frac{E_{0} \times B_{0}}{\mu_{0}}
$$

The magnitude of amplitude of the Poynting vector can be represented by

$$
\mathcal{P}_{0}=c \varepsilon_{0} E_{0}^{2}=\frac{1}{\mu_{0}} c B_{0}^{2}=\frac{1}{2} c\left(\varepsilon_{0} E_{0}^{2}+\frac{1}{\mu_{0}} B_{0}^{2}\right)
$$

This formula also indicates the quantitative relation between the electric field strength vector and the magnetic induction strength vector.

\subsection{Wave-Train Length of a Single Linearly Polarized Photon}

It is not difficult to imagine that the envelope of a single photon expressing in the plane wave functions (7-1) and (7-2) is a cuboid. So, the cross-section of the photon is even. That is to say, the cross-sectional area on the same photon is equal everywhere.

The single-photon energy $\mathcal{E}$ can represent by integrating the Poynting vector $\mathcal{P}_{0}$, the cross-sectional area $\sigma_{P}$, and the time taken for the wave-train passing through a position $z=0$.

$$
\begin{aligned}
\mathcal{E} & =\int_{0}^{\mathcal{T}} \boldsymbol{\sigma}_{P} \cdot \mathcal{P} \mathrm{d} t \\
& =\int_{0}^{\mathcal{T}}\left(\sigma_{P} \boldsymbol{e}_{z}\right) \cdot\left(\mathcal{P}_{0} \sin ^{2} \omega t \boldsymbol{e}_{z}\right) \mathrm{d} t \\
& =\sigma_{p} \mathcal{P}_{0} \int_{0}^{\mathcal{T}} \sin ^{2} \omega t \mathrm{~d} t \\
& =\frac{1}{2} \sigma_{P} \mathcal{P}_{0} \int_{0}^{\mathcal{T}}(1-\cos 2 \omega t) \mathrm{d} t \\
& =\frac{1}{2} \sigma_{p} \mathcal{P}_{0}\left(\mathcal{T}-\frac{\sin 2 \omega \mathcal{T}}{2 \omega}\right)
\end{aligned}
$$

where $\sigma_{P}=\sigma_{P} \boldsymbol{e}_{z}$ and Equation (14) are employed.

Since a single photon is a complete quantum, $\mathcal{T}$ must be constrained to the quantization conditions. What conditions can meet the requirements of a single photon for $\mathcal{T}$ ? The following steps can take for derivation: 
First, the photon energy does not depend on the sine function of the angular frequency. Thus, $2 \omega \mathcal{T}=N \pi$ can be derived, so can obtain $\mathcal{T}=N T / 4$.

Second, since a single photon is the minimum unit of light, $N$ can only be 1,2 , 3,4 .

Third, because the duration of a photon passing through a position, for example, $z=0$, is an integer multiple of the period $T$ instead of a half-integer multiple of period $T$. That is to say, the wave-train length of the photon must be an integer multiple of wavelength instead of a half-integer multiple of the wavelength. So the only value of $N$ is 4 . Applying the Fourier series expansion of the wave function (7-1) can prove this point.

It implies that the oscillating duration of a photon at a place equals the period of the photon

$$
\mathcal{T}=T
$$

The wave-train length of a photon equals the wavelength of the photon

$$
\mathcal{L}=c T=\lambda
$$

Substituting $\mathcal{T}=T$ into the Equation (17-e) can obtain the photon energy $\mathcal{E}$ expressed in terms of the cross-sectional area $\sigma_{P}$, the magnitude of the Poynting vector amplitude $\mathcal{P}_{0}$, and the period $T$.

$$
\mathcal{E}=\frac{1}{2} \sigma_{P} \mathcal{P}_{0} T=\hbar \omega
$$

The equation contains two unknowns: $\sigma_{P}$ and $\mathcal{P}_{0}$. To finding the crosssectional area $\sigma_{P}$, it is necessary to find the magnitude of the Poynting vector amplitude, $\mathcal{P}_{0}$.

\subsection{Analysis of Compton Scattering from the Viewpoint of Classical Mechanics}

When a free electron encounters an incident photon at $z=0$, the electron is affected by the photon's electric field, and its motion state is changed (Figure 2).

Suppose that the incident photon propagates along the $z$-axis, the electric field oscillates along the $x$-axis. After the photon interacts with the electron, its direction of propagation changes by an angle $\theta$ turns to direction $\boldsymbol{p}^{\prime}$. The electric field strength vector of photon changes from $\boldsymbol{E}$ to $\boldsymbol{E}^{\prime \prime}$, also changes angle $\theta$ synchronously.

Ignoring the numerical difference between $\left|\boldsymbol{E}^{\prime \prime}\right|$ and $|\boldsymbol{E}|$, the effect of the changing electric field strength vector acting on the electron is equivalent to that of the electric field strength vector $\boldsymbol{E}^{\prime}$, whose direction is close to the angular bisector between $\boldsymbol{E}$ and $\boldsymbol{E}^{\prime \prime}$.

The components of the equivalent electric field strength $\boldsymbol{E}^{\prime}$ on the $x$-axis and the $z$-axis are respectively

$$
\left\{\begin{array}{l}
E_{x}^{\prime}=E^{\prime} \cos \frac{\theta}{2} \\
E_{z}^{\prime}=-E^{\prime} \sin \frac{\theta}{2}
\end{array}\right.
$$




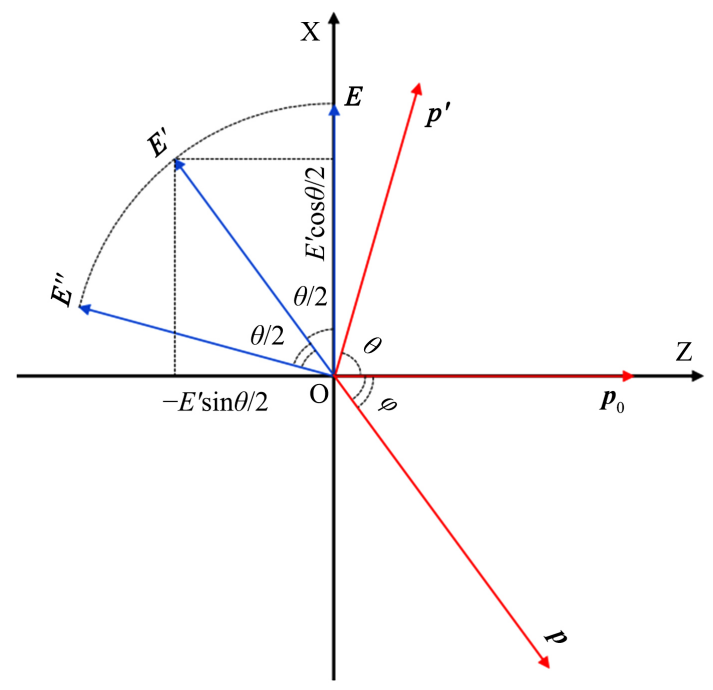

Figure 2. The change of the electric field strength vector and the momentum vector of a photon.

Assuming that the electron is initially at $z=0$, applying the Equation (7-1) can obtain the following results

$$
\left\{\begin{array}{l}
E_{x}^{\prime}=E_{0} \cos \frac{\theta}{2} \sin \omega t \\
E_{z}^{\prime}=-E_{0} \sin \frac{\theta}{2} \sin \omega t
\end{array}\right.
$$

Let the charge of the electron be $-e$, then the components of the electric field force acting on the electron are respectively

$$
\left\{\begin{array}{l}
F_{x}=m \ddot{x}=-e E_{x}^{\prime}=-e E_{0} \cos \frac{\theta}{2} \sin \omega t \\
F_{z}=m \ddot{z}=-e E_{z}^{\prime}=e E_{0} \sin \frac{\theta}{2} \sin \omega t
\end{array}\right.
$$

Thus, the acceleration components of the electron motion are given by

$$
\left\{\begin{array}{l}
\ddot{x}=-\frac{e E_{0}}{m} \cos \frac{\theta}{2} \sin \omega t \\
\ddot{z}=\frac{e E_{0}}{m} \sin \frac{\theta}{2} \sin \omega t
\end{array}\right.
$$

Assuming that the initial velocity $\dot{x}_{0}=0, \dot{z}_{0}=0$; the initial displacement $x_{0}=0, z_{0}=0$; the interaction time is $t=\tau$. Integrating $\ddot{x}$ and $\ddot{z}$ about $t$ from 0 to $\tau$, the velocity components of the electron along the $x$-axis and $z$-axis are respectively

$$
\left\{\begin{array}{l}
\dot{x}_{\tau}=-\frac{e E_{0}}{m} \cos \frac{\theta}{2} \int_{0}^{\tau} \sin \omega t \mathrm{~d} t=-\frac{e E_{0}}{m \omega} \cos \frac{\theta}{2}(1-\cos \omega \tau) \\
\dot{z}_{\tau}=\frac{e E_{0}}{m} \sin \frac{\theta}{2} \int_{0}^{\tau} \sin \omega t \mathrm{~d} t=\frac{e E_{0}}{m \omega} \sin \frac{\theta}{2}(1-\cos \omega \tau)
\end{array}\right.
$$

The displacement components of the electron along the $\mathrm{x}$-axis and $\mathrm{z}$-axis are respectively 


$$
\left\{\begin{array}{l}
x_{\tau}=-\frac{e E_{0}}{m} \cos \frac{\theta}{2} \int_{0}^{\tau} \int_{0}^{t} \sin \omega t^{\prime} \mathrm{d} t^{\prime} \mathrm{d} t=-\frac{e E_{0}}{m \omega} \cos \frac{\theta}{2}\left(\tau-\frac{\sin \omega \tau}{\omega}\right) \\
z_{\tau}=\frac{e E_{0}}{m} \sin \frac{\theta}{2} \int_{0}^{\tau} \int_{0}^{t} \sin \omega t^{\prime} \mathrm{d} t^{\prime} \mathrm{d} t=\frac{e E_{0}}{m \omega} \sin \frac{\theta}{2}\left(\tau-\frac{\sin \omega \tau}{\omega}\right)
\end{array}\right.
$$

In the above formulas, the interaction time $\tau$ between photon and electron does not exceed the photon period $T$.

In the case that the electric field of a photon is wide enough, the electron can move in the electric field for a complete period, that is, $\tau=T$, so the velocity components of the electron along the $x$-axis and $z$-axis are given by the following formulas

$$
\left\{\begin{array}{l}
\dot{x}_{T}=-\frac{e E_{0}}{m \omega} \cos \frac{\theta}{2}(1-\cos \omega T)=0 \\
\dot{z}_{T}=\frac{e E_{0}}{m \omega} \sin \frac{\theta}{2}(1-\cos \omega T)=0
\end{array}\right.
$$

In other words, the velocity of the free electron is zero after the electric field force of the photon acts for a period $T$; that is to say, the electron velocity returns to that of the initial state.

It can be seen from Equations (26-1) (26-2) that if $\tau=T$, the displacement components of electron along the $x$-axis and $z$-axis are

$$
\left\{\begin{array}{l}
x_{T}=-\frac{e E_{0}}{m \omega} T \cos \frac{\theta}{2}=-\frac{2 \pi e E_{0}}{m \omega^{2}} \cos \frac{\theta}{2} \\
z_{T}=\frac{e E_{0}}{m \omega} T \sin \frac{\theta}{2}=\frac{2 \pi e E_{0}}{m \omega^{2}} \sin \frac{\theta}{2}
\end{array}\right.
$$

where the relationship $T=2 \pi / \omega$ is employed.

The magnitude of electron displacement is given by

$$
s_{T}=\sqrt{x_{T}^{2}+z_{T}^{2}}=\frac{2 \pi e E_{0}}{m \omega^{2}}
$$

In the above formulas, the electric field strength vector $E_{0}$ of the photon is involved, which is an unknown quantity.

In the case that the electric field of the photon is narrow enough that the electron ejects from the electric field within the time $T$; thus, the momentum components of the electron along the $x$-axis and $z$-axis are respectively

$$
\left\{\begin{array}{l}
p_{x}=m \dot{x}=-\frac{e E_{0}}{\omega}(1-\cos \omega \tau) \cos \frac{\theta}{2} \\
p_{z}=m \dot{z}=\frac{e E_{0}}{\omega}(1-\cos \omega \tau) \sin \frac{\theta}{2}
\end{array}\right.
$$

The recoiling angle $\varphi$ of the electron can express as the following formula

$$
\operatorname{tg} \varphi=\left|\frac{p_{x}}{p_{z}}\right|=\operatorname{ctg} \frac{\theta}{2}
$$

This relationship is consistent with the Compton scattering Equation (2-2) under the approximate condition $\kappa \ll 1$. The following angular relationship can derive from Equation (31) 


$$
\varphi=\frac{1}{2}(\pi-\theta)
$$

It implies that the electron recoil direction is opposite to that of the electric field vector $\boldsymbol{E}^{\prime}$ under the condition $\kappa \ll 1$.

The kinetic energy of the recoil electron can be given by

$$
\begin{aligned}
\Delta \mathcal{E} & =\frac{1}{2} m\left(\dot{x}^{2}+\dot{z}^{2}\right) \\
& =\frac{2 e^{2} E_{0}^{2}}{m \omega^{2}} \sin ^{4} \frac{\omega \tau}{2}
\end{aligned}
$$

Under the condition $\kappa \ll 1$, Equation (6) becomes

$$
\begin{aligned}
\Delta \mathcal{E} & =\frac{2(\hbar \omega)^{2}}{m c^{2}} \cos ^{2} \varphi \\
& =\frac{2(\hbar \omega)^{2}}{m c^{2}} \sin ^{2} \frac{\theta}{2}
\end{aligned}
$$

Comparing the Equation (30) with (5) can obtain the following equations under the approximate condition $\kappa \ll 1$

$$
\left\{\begin{array}{l}
-\frac{e E_{0}}{\omega}(1-\cos \omega \tau) \cos \frac{\theta}{2}=-\frac{2 \hbar \omega}{c} \cos \varphi \sin \varphi \\
\frac{e E_{0}}{\omega}(1-\cos \omega \tau) \sin \frac{\theta}{2}=\frac{2 \hbar \omega}{c} \cos ^{2} \varphi
\end{array}\right.
$$

In Equations (35-1) (35-2), there are two types of physical quantities: one is related to the photon itself and natural constants, such as $e, E_{0}, \hbar$, and $\omega$; the other is the variables related to the interaction process, such as $\tau, \theta$, and $\varphi$. By comparing the Equation (33) with (34), then applying the method of separating variables can obtain the solutions to the above equations

$$
\left\{\begin{array}{l}
\frac{e E_{0}}{\omega}=\frac{\hbar \omega}{c} \\
\sin ^{2} \frac{\omega \tau}{2}=\sin \frac{\theta}{2}=\cos \varphi
\end{array}\right.
$$

Equation (36-1) represents the relationship between electric field strength and angular frequency of the photon; Equation (36-2) represents the correlation that the interaction time $\tau$, the scattering angle $\theta$ of the photon, and the recoiling angle $\varphi$ of the electron.

\subsection{Formulas for the Size and Shape of a Single Photon}

Equation (36-1) can further transform into the following form

$$
E_{0}=\frac{\hbar \omega^{2}}{c e}
$$

Substituting Equation (37) into Equation (16) can obtain the magnitude of amplitude of the Poynting vector

$$
\mathcal{P}_{0}=\varepsilon_{0} c E_{0}^{2}=\frac{\hbar \omega^{4}}{4 \pi \alpha c^{2}}
$$


where

$$
\alpha=\frac{e^{2}}{4 \pi \varepsilon_{0} \hbar c}
$$

is the fine structure constant.

Equation (38) implies that the Poynting vector amplitude is directly proportional to the fourth power of angular frequency.

Employing $T=2 \pi / \omega$ and $\lambda=c T$ and substituting Equation (38) into (20) can obtain a formula

$$
\sigma_{P}=\frac{\alpha}{\pi^{2}} \lambda^{2}
$$

It is the formula for the cross-sectional area of a single linearly polarized photon. It is evident from this formula that the cross-sectional area of a single photon is directly proportional to the square of the photon wavelength, and the proportional coefficient is $\alpha / \pi^{2}=7.394 \times 10^{-4}$.

So, what are the photon lengths in the direction of the electric field and magnetic field?

The Equation (34-c) can transform into the following form

$$
\begin{aligned}
\Delta \mathcal{E} & =\frac{2(\hbar \omega)^{2}}{m c^{2}} \cos ^{2} \varphi \\
& =\frac{2 r_{e} \cdot(2 \lambda / \pi)}{\left(\alpha / \pi^{2}\right) \lambda^{2}} \cdot \cos ^{2} \varphi \cdot \hbar \omega
\end{aligned}
$$

where

$$
r_{e}=\frac{1}{4 \pi \varepsilon_{0}} \frac{e^{2}}{m c^{2}}
$$

is the classical electron radius, $2 r_{e}$ is the classical electron diameter, $\left(\alpha / \pi^{2}\right) \lambda^{2}$ in the denominator is the cross-sectional area of the photon, the product

$2 r_{e}(2 \lambda / \pi)$ in the numerator represents the maximum area swept by the electron in the electric field of the photon; thus, the factor $2 \lambda / \pi$ represents the maximum displacement of the electron in the direction of the electric field of the photon. Therefore, the factor $2 \lambda / \pi$ is the length of the photon in the direction of the electric field

$$
\mathcal{L}_{E}=\frac{2}{\pi} \lambda
$$

Since this quantity is independent of the position on the $y$-axis, it is correct that the cross-section of the photon is a rectangle. The length of the photon in the magnetic field direction is

$$
\mathcal{L}_{M}=\frac{\alpha}{2 \pi} \lambda
$$

where the Equation (40) for the cross-sectional area is employed. The expression for photon's volume is 


$$
V_{P}=\frac{\alpha}{\pi^{2}} \lambda^{3}
$$

These formulas for the size and shape of a single photon only contain one physical quantity of photon-the wavelength $\lambda$, and one physical constant-the fine structure constant $\alpha$.

\subsection{The Energy Density of a Single Photon}

After the volume formula (45) of the photon establishment, the average energy density of the photon is given by

$$
\bar{u}=\frac{\hbar \omega}{V_{P}}=\frac{\hbar \omega^{4}}{8 \pi \alpha c^{3}}
$$

This formula shows that a single photon's energy density is directly proportional to the fourth power of its angular frequency.

\subsection{The Structure Model of a Linearly Polarized Photon}

The envelope and the electromagnetic field strength of a single photon are two different concepts. According to wave functions (7-1) (7-2), the electromagnetic field strength changes with time and position, but the envelope of electromagnetic wave propagation in the vacuum remains unchanged.

The ratio of photon thickness to photon length is $\mathcal{L}_{M} / \mathcal{L}=\alpha / 2 \pi=1.16 \times 10^{-3}$, so the photon thickness is very thin.

The structural model of linearly polarized photons is shown in Figure 3.

\section{Electromagnetic Mechanism of Compton Scattering}

\subsection{Explanation of Compton Effect by Classical Electrodynamics}

Why must Compton scattering experiment employ X-ray and $\gamma$-ray rather than visible light? Explaining this issue needs the structure model of a single photon besides the theory of atomic structure.

As shown in Figure 4 is a cross-section of a single photon that the origin of coordinates is at its center.

How does the Compton effect generate? Discussion on this problem can be in two cases according to the interaction time $\tau$.

1) $\tau=T$

It refers to the case, the electric field of photon always acts on the electron throughout the period $T$. The displacement of the electron reaches the maximum value after the time $\tau=T$.

Substituting Equation (36-1) into Equation (29) can obtain the electron displacement:

$$
s_{T}=\frac{2 \pi e E_{0}}{m \omega^{2}}=\frac{2 \pi \hbar}{m c}=\frac{h}{m c}=\lambda_{C}
$$

This formula shows that the maximum displacement of the electron in the electric field is the Compton wavelength if the interaction time between photon and electron is period $T$; that is to say, $|s|_{\max }=\lambda_{C}$. 


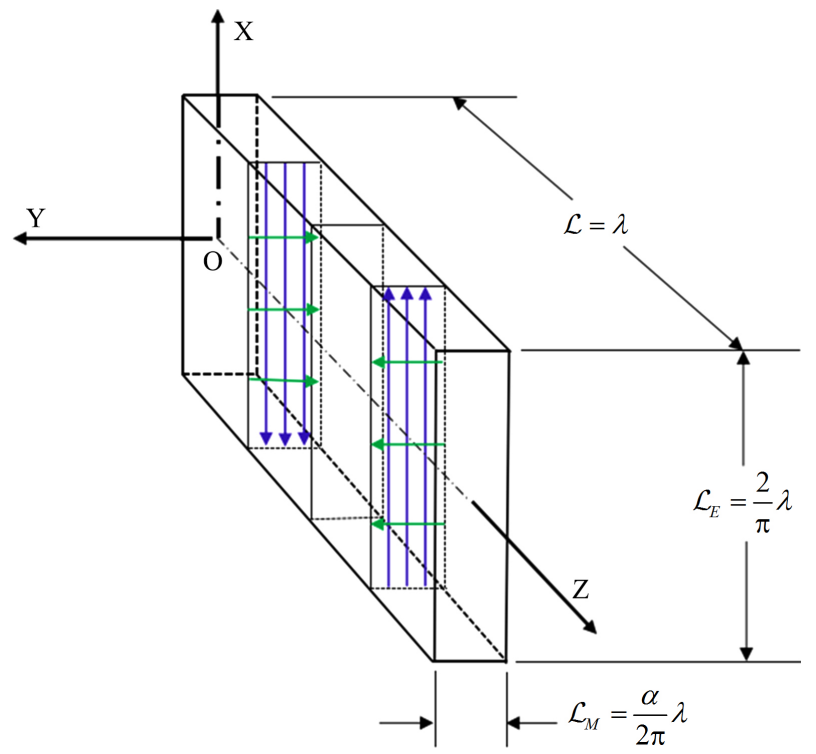

Figure 3. This diagram shows the electromagnetic field structure of a single linearly polarized photon, in which the blue arrows indicate the direction of the electric field strength, and the green arrows indicate the direction of the magnetic induction strength.

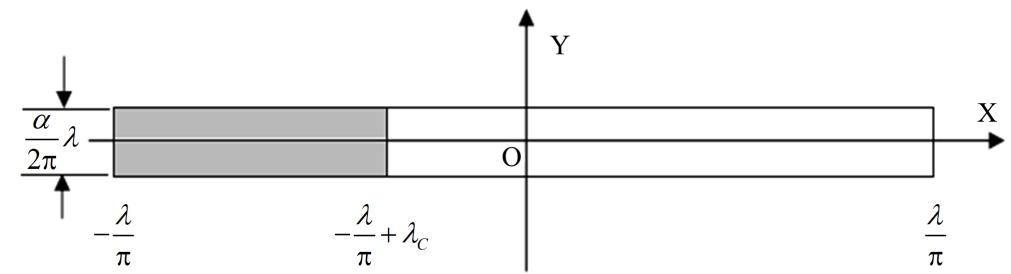

Figure 4. This diagram shows the cross-sectional view of a single linearly polarized photon. The electron colliding with the shadow part of the photon ejects from the electromagnetic field resulting in the Compton effect.

Therefore, it is necessary to add a new explanation to Compton scattering theory:

Compton wavelength is the maximum displacement of the electron in enough wide electric field of a single photon.

Substituting $\tau=T$ and Equation (36-1) into Equation (33-b), the formula of the kinetic energy of the electron becomes

$$
\Delta \mathcal{E}_{T}=\frac{2(\hbar \omega)^{2}}{m c^{2}} \sin ^{4} \frac{\omega T}{2}=0
$$

This equation implies that a photon collides with an electron if the interaction time reaches the period $T$, the photon will bypass the electron without any obstruction. The electron returns to rest after displacement $\lambda_{C}$, and it does not get the kinetic energy from the photon. The propagation direction of the photon does not change. In this case, there is no Compton effect.

The case that the electron moved $\lambda_{C}$ in the negative direction of the $x$-axis in the electric field of the photon without being ejected must satisfy the follow- 
ing two conditions at the same time:

a) The photon electric field is wide enough that its width satisfies $2 \lambda / \pi \geq \lambda_{C}$; that is, $\lambda \geq \pi \lambda_{C} / 2$.

b) In collision, the electron must fall within the interval $-\left(\lambda / \pi-\lambda_{C}\right) \rightarrow \lambda / \pi$ of the $x$-axis.

2) $\tau<T$

It refers to the case the electron is ejected from the field so that the electron displacement can not attain $\lambda_{C}$ when the interaction has not yet reached the period $T$.

In this way, if $\tau<T$, the electron gains kinetic energy from the electric field of the photon, so Equation (33-b) becomes

$$
\Delta \mathcal{E}=\frac{2(\hbar \omega)^{2}}{m c^{2}} \sin ^{4} \frac{\omega \tau}{2}>0
$$

The photon loses the corresponding energy and becomes longer in wavelength simultaneously, which is the Compton effect.

For this phenomenon to occur, one of the following two conditions must be satisfied:

a) The electric field of the photon is so narrow that its width satisfies $2 \lambda / \pi<\lambda_{C}$, i.e., $\lambda<\pi \lambda_{C} / 2$.

b) The electron falls in the interval $-\lambda / \pi \rightarrow-\left(\lambda / \pi-\lambda_{C}\right)$ of the $x$-axis, although the photon electric field is wide enough to satisfy the condition $\lambda \geq \pi \lambda_{C} / 2$.

In the cases 1) $\tau=T$ and 2) $\tau<T$, the electron displacement in the electric field can only be the smaller one of $\lambda_{C}$ and $2 \lambda / \pi$ or expressed by the following formula:

$$
|s|_{\max }=\min \left\{2 \lambda / \pi, \lambda_{C}\right\}
$$

Compton scattering is due to the electron is ejected from the electric field when a photon collides with an electron and the interaction time does not reach period $\mathrm{T}$.

\subsection{The Probability of Compton Effect When Photon Collides with Electron}

Under the condition $\lambda \geq \pi \lambda_{C} / 2$, when a photon collides with an electron, there is a certain probability that the electron will fall into the region $-\lambda / \pi \rightarrow-\left(\lambda / \pi-\lambda_{C}\right)$ in the electric field, resulting in the Compton effect. There is a certain probability that the electron will fall into the region $-\left(\lambda / \pi-\lambda_{C}\right) \rightarrow+\lambda / \pi$ in the electric field without the Compton effect. The occurrence probability of the Compton effect is given by

$$
p=\frac{\lambda_{C}}{2 \lambda / \pi}=\frac{\pi \lambda_{C}}{2 \lambda}
$$

Under the condition $\lambda<\pi \lambda_{C} / 2$, the occurrence probability of the Compton effect is given by 


$$
P=1
$$

It means that the Compton effect will inevitably occur when the photon collides with the electron if $\lambda<\pi \lambda_{C} / 2$.

Taken together, when a photon collides with an electron, the probability of the Compton effect can be expressed as

$$
p= \begin{cases}\frac{\pi \lambda_{C}}{2 \lambda} & \left(\text { if } \lambda \geq \frac{\pi \lambda_{C}}{2}\right) \\ 1 & \left(\text { if } \lambda<\frac{\pi \lambda_{C}}{2}\right)\end{cases}
$$

It is evidently from the above formula:

1) When a photon satisfying the condition $\lambda \geq \pi \lambda_{C} / 2$ collides with a free electron, there is always a certain probability of the Compton effect, which value is inversely proportional to the wavelength. Especially for a photon satisfying the condition $\lambda \gg \pi \lambda_{C} / 2$, the Compton effect hardly appears.

2) When a photon satisfying the condition $\lambda<\pi \lambda_{C} / 2$ collides with an electron, the Compton effect always occurs.

However, if the photon wavelength is close to or smaller than the Compton wavelength, the electron cannot be regarded as a point-like particle. This situation is beyond the scope of the present article.

\section{The Structure of a Single Circularly Polarized Photon}

\subsection{The Spin of a Single Circularly Polarized Photon}

For a single circularly polarized photon, the following functions can express the electric field strength and magnetic induction strength:

$$
\left\{\begin{array}{l}
\boldsymbol{E}=E_{0}\left[\sin \omega\left(t-\frac{z}{c}\right) \boldsymbol{e}_{x} \mp \cos \omega\left(t-\frac{z}{c}\right) \boldsymbol{e}_{y}\right] \\
\boldsymbol{B}=B_{0}\left[ \pm \cos \omega\left(t-\frac{z}{c}\right) \boldsymbol{e}_{x}+\sin \omega\left(t-\frac{z}{c}\right) \boldsymbol{e}_{y}\right]
\end{array}\right.
$$

where the sign “ $\mp$ ” in Equation (53-1) corresponding to “ \pm ” in Equation (53-2) represents the right-handed photon and the left-handed photon.

Similarly to the linearly polarized photon, the relationship between electric field strength $E$ and vector potential $A$ conforms to the following relationship

$$
\boldsymbol{E}=-\frac{\partial \boldsymbol{A}}{\partial t}
$$

By integrating $E$ over $t$ can obtain the expression of the vector potential

$$
\boldsymbol{A}=-\int \boldsymbol{E} \mathrm{d} t=\frac{E_{0}}{\omega}\left[\cos \omega\left(t-\frac{z}{c}\right) \boldsymbol{e}_{x} \pm \sin \omega\left(t-\frac{z}{c}\right) \boldsymbol{e}_{y}\right]
$$

Comparing Equation (55) with (53-2) can obtain

$$
\boldsymbol{B}= \pm \omega \frac{B_{0}}{E_{0}} \boldsymbol{A}
$$

Employing the relationship between the amplitude of electric field strength 
and amplitude of magnetic induction strength, $B_{0}=E_{0} / c$, can obtain

$$
\boldsymbol{B}= \pm \frac{\omega}{c} \boldsymbol{A}
$$

It conforms to the relationship $\boldsymbol{B}=\nabla \times \boldsymbol{A}$.

The Poynting vector of the photon is

$$
\begin{aligned}
\mathcal{P} & =\frac{1}{\mu_{0}} \boldsymbol{E} \times \boldsymbol{B} \\
& =\frac{1}{\mu_{0}} \boldsymbol{E} \times\left( \pm \frac{\omega}{c} \boldsymbol{A}\right) \\
& = \pm \frac{\omega}{c \mu_{0}} \boldsymbol{E} \times \boldsymbol{A} \\
& = \pm \omega\left(c \varepsilon_{0} \boldsymbol{E} \times \boldsymbol{A}\right) \\
& = \pm \boldsymbol{s} \omega
\end{aligned}
$$

where $c^{2}=1 / \varepsilon_{0} \mu_{0}$ is employed.

A spin angular momentum flow vector appears in the formula.

$$
\boldsymbol{s}=c \varepsilon_{0} \boldsymbol{E} \times \boldsymbol{A}= \pm \frac{\mathcal{P}}{\omega}
$$

This formula can also express as

$$
\mathcal{P}= \pm s \omega
$$

Multiply the cross-sectional area $\sigma_{P}=\sigma_{P} \boldsymbol{e}_{z}$ of the photon on both sides of the equation, and then integrate the two sides over time $t$ from 0 to $T$, and the result is equal to photon energy.

The left side of the equation is given by

$$
\mathcal{E}=\int_{0}^{T} \mathcal{P} \cdot\left(\sigma_{P} \boldsymbol{e}_{z}\right) \mathrm{d} t=\hbar \omega
$$

The right side of the equation is given by

$$
\mathcal{E}= \pm \int_{0}^{T}(\boldsymbol{s} \omega) \cdot\left(\sigma_{P} \boldsymbol{e}_{z}\right) \mathrm{d} t= \pm \mathcal{S} \omega
$$

where $\sigma_{P}$ is the cross-sectional area of the photon entity part. Comparing the left and right sides of the equation can obtain that the spin angular momentum of the photon is as the following formula:

$$
\mathcal{S}= \pm \hbar
$$

The Poynting vector is always along the photon propagation direction. The directions of spin angular momentum of the right-handed photon and the lefthanded photon are opposite.

Taken together, it can conclude that the spin angular momentum of a linearly polarized photon is 0 , but the angular momentum of a circularly polarized photon is $\pm \hbar$.

\subsection{Electromagnetic Structure of Single Circularly Polarized Photon}

In this section, use the Equations (53-1) and (53-2) to replace the Equations 
(7-1) and (7-2) to derive some corresponding formulas of the circularly polarized photon following the derivation process of the linearly polarized photon.

First of all, the photon length $\mathcal{L}=\lambda$ should be acceptable.

Similarly to the derivation of the Equation (37), the magnitude of amplitude of the electric field strength vector is given by

$$
E_{0}=\frac{\hbar \omega^{2}}{c e}
$$

Applying the wave functions (53-1) (53-2) can obtain that the Poynting vector is stable, its direction is always along the propagation direction of the photon, and its magnitude is given by

$$
\mathcal{P}=\frac{\hbar \omega^{2}}{4 \pi \alpha c^{2}}
$$

Thereby obtain the cross-sectional area of the photon entity part

$$
\sigma_{p}=\frac{\hbar \omega}{\mathcal{P}}=\frac{\alpha}{2 \pi^{2}} \lambda^{2}
$$

Similar to the derivation of the Equations (41) and (43), the maximum displacement of recoil electron in photon electric field is $\lambda / \pi$, which can write as radius due to its rotational symmetry.

$$
R=\frac{\lambda}{2 \pi}
$$

If regarding the photon as a rigid body with rotational symmetry, the moment of inertia is

$$
I=\eta m_{p} R^{2}
$$

where $\eta$ is a presupposed coefficient, depending on the rigid body shape and how to rotate around the axis; $m_{p}$ is the photon mass whose value is $\hbar \omega / c^{2}$.

Because the spin angular momentum of a photon is $|\mathcal{S}|=\hbar$, it can express by the product of the moment of inertia and rotation angular speed

$$
|\mathcal{S}|=I \omega=\eta m_{p} R^{2} \omega=\eta \frac{\hbar \omega^{2}}{c^{2}}\left(\frac{\lambda}{2 \pi}\right)^{2}=\hbar
$$

Thereby obtain that

$$
\eta=1
$$

That is to say, the moment of inertia of a photon as a rigid body is given by

$$
I=m_{p} R^{2}
$$

What kind of rigid body has such an expression of the moment of inertia? If considering the symmetry and the length $\mathcal{L}=\lambda$, the rigid body can only be a hollow cylinder, which looks like a round pipe.

The radius of the hollow cylinder is $R=\lambda / 2 \pi$, the cross-sectional area of the entity part is $\sigma_{p}=\alpha \lambda^{2} / 2 \pi^{2}$, and the difference between the inner radius and the outer radius of the cylinder is

$$
\delta=\frac{\alpha}{2 \pi^{2}} \lambda
$$


It is the thickness of the shell of the circular cylinder containing the electromagnetic field. Compared with the cylinder radius, this thickness is insignificant. The ratio of the thickness to the radius is $\delta / R=\alpha / \pi=2.32 \times 10^{-3}$, so can ignore $\delta$ when calculating the moment of inertia, but the photon energy concentrates in this shell.

As shown in Figure 5 is a cross-section of a circularly polarized photon. As shown in Figure 6 is the electromagnetic field structure of the circularly polarized photon.

The linearly polarized photon is a cuboid entity; the circularly polarized photon is hollow, the energy concentrates in the entity part that is a membrane-like shell of a cylinder. This conclusion is a bit unexpected.

Figure 7 shows cross-sectional views of right-handed photon and left-handed photon, and the phase difference between two adjacent cross-sectional views is $\pi / 2$.

The formulas for photon volume, energy density, and Compton effect probability obtain easily, omit here.

\section{Discussion and Summary}

\subsection{Several Photon Models Worthy of Discussion}

Scholars have published theories of various photon models since Planck proposed the concept of quantum in 1900. All these models agree that photons are circularly polarized. Here are five examples published in recent years. The wave functions of the electric field strength and magnetic field induction strength of circularly polarized photon [3] are exact (53-1) (53-2), but the values about the wave-train length and cross-sectional area of the photon proposed by scholars are different.

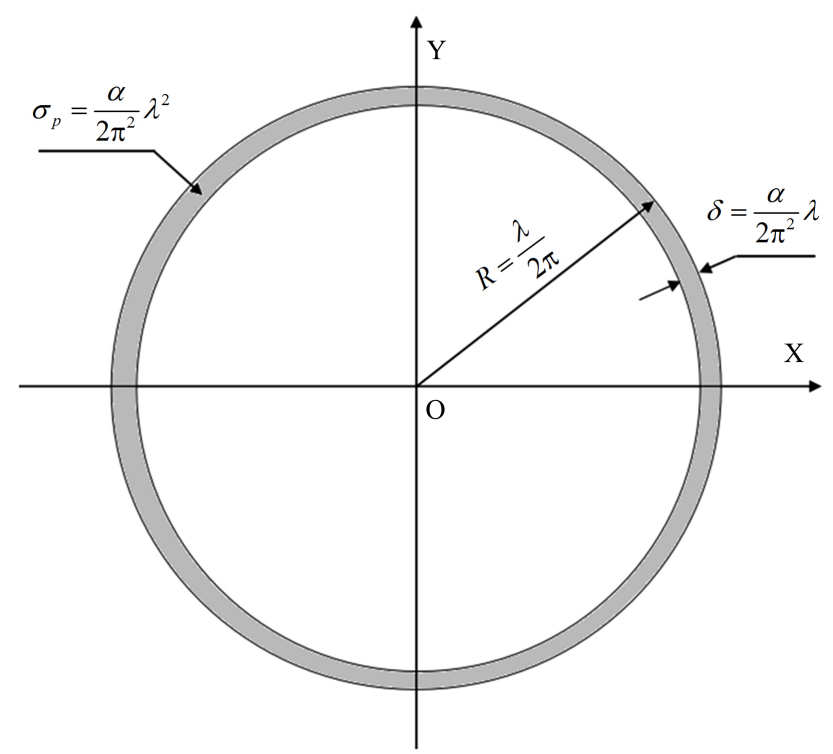

Figure 5. This diagram shows a cross-section of a circularly polarized photon, where the entity part (shaded part) is so exaggerated as to seeing the electromagnetic field structure. 


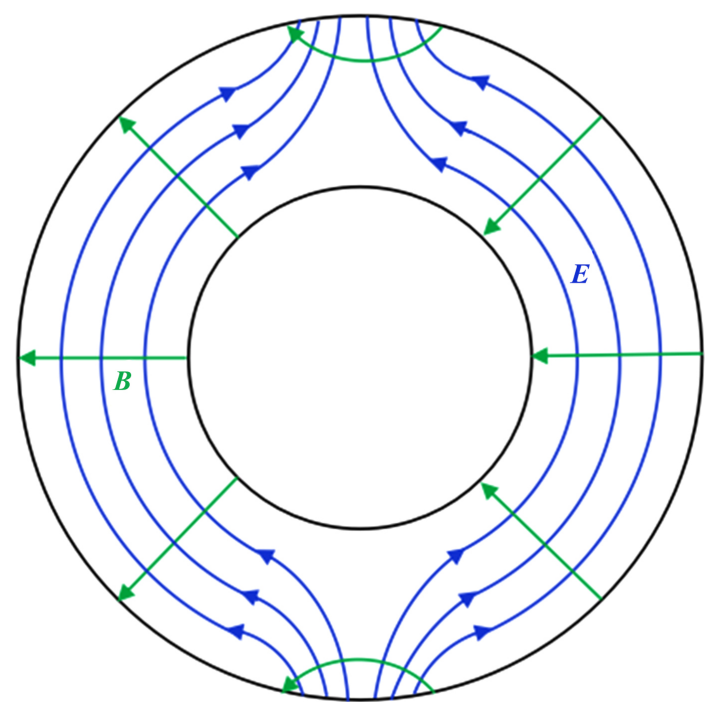

Figure 6. This diagram shows the electromagnetic field structure of the circularly polarized photon, where the entity part is so exaggerated as to seeing the electromagnetic field structure. The blue arrows indicate the direction of electric field strength, and the green arrows indicate the direction of magnetic induction strength.
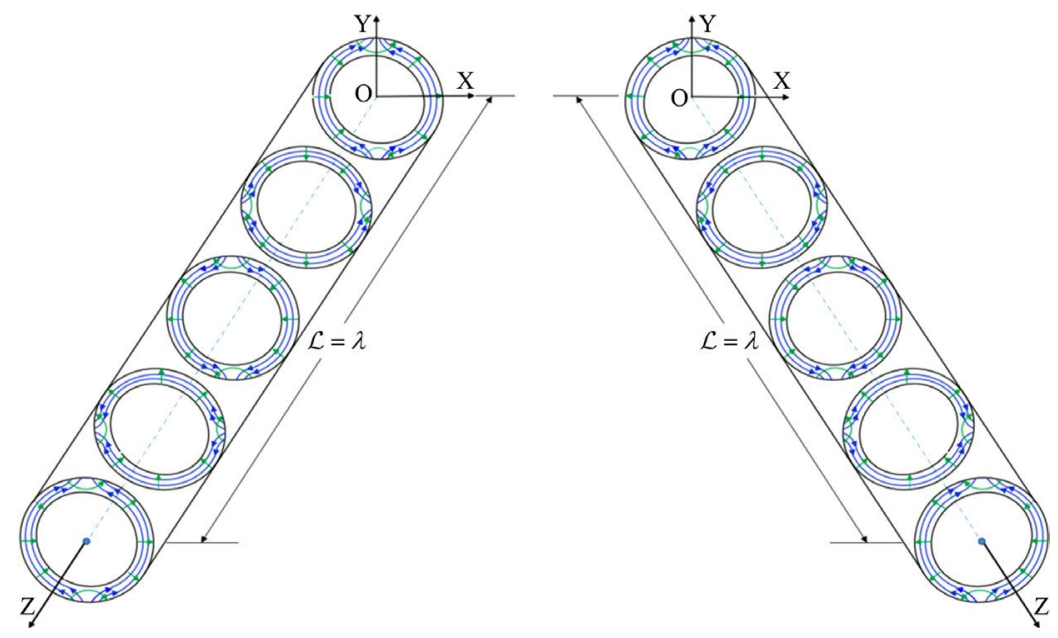

Figure 7. This set of diagrams show the electromagnetic field structure of the circularly polarized photon. The blue arrows indicate the direction of electric field strength, and the green arrows indicate the direction of magnetic induction strength. The left one represents the right-handed photon, and the right one represents the left-handed photon.

Example 1: Circular ellipsoid model [4]

Geoffrey Hunter, Marian Kowalski, and Camil Alexandrescu imitated Bohr's hydrogen atom model and put forward a circular ellipsoid model of the circular polarization states. According to this model, the photon length in the propagation direction is equal to the wavelength $\lambda$, and the radius of the cross-section at the strongest electromagnetic field is $R=\lambda / 2 \pi$. The electric field strength increases in proportional to the radius $r$ within the radius $R$ and decreases grad- 
ually outside the radius $R$ in inverse proportion to the radius $r$. Therefore, the electromagnetic field is an evanescent wave.

Example 2: Spherical spiral wave packet model [5]

Dong-Lin $\mathrm{Zu}$ proposed a spherical model theory of photon, whose main view is to treat a photon as a sphere, which rotates around the $z$-axis of the propagation direction, and the angular momentum is the reduced Planck constant $\hbar$. Thus, a single photon radius is $r_{p}=\sqrt{5 / 2} \lambda$. So, the oscillation duration is $\mathcal{T}=2 \sqrt{5 / 2} T$.

Example 3: Multi-wavelength cylindrical spiral wave packet model [6]

Herbert Weidner proposed a cylindrical model based on the hypothesis that a single photon is a circularly polarized electromagnetic wave packet with a spin angular momentum $\hbar$ and energy $\hbar \omega$. The author deduced that the radius of a single photon is approximately $r_{p}=(3 \sqrt{2} / 4 \pi) \lambda \approx 0.338 \lambda$. Using Heisenberg's uncertainty principle, the author estimated that the photon length is at least about $100,000 \lambda$.

Example 4: Circular coin model [7]

Sen Nian Chen proposed a wave packet model based on the Maxwell theory and the principle of electric charges quantization. The author proved that the photon is circularly polarized and the shape like a circular coin, whose length of propagation direction (coin thickness) is $\mathcal{L}=\pi \alpha \lambda / 2$, the maximum value of circle radius (coin radius) is $R_{\max }=\sqrt{3} \lambda / 2 \pi$. The author also proved that the circularly polarized electromagnetic field is wrapped by a cylindrical lateral membrane with charge $\pm e$.

Example 5: Half-wavelength cylindrical model [8]

Shan-Liang Liu proposed a new cylindrical model. The author deduced mathematically that the longitudinal interval of a single photon is equal to half a wavelength in space and half a cycle in time; that is to say, the wave-train length is $\mathcal{L}=\lambda / 2$, the duration at a point is $\mathcal{T}=T / 2$.

According to the principle of atomic ionization by photon electromagnetic field, the author deduced the vector potential of photon electromagnetic field and obtained that the radius of photon cylinder is $r_{p}=2 \sqrt{2 r_{e} \lambda} /(\sqrt{2}-1) \pi$.

The theoretical analysis of the above models shows that these models have the following troubles:

Trouble 1-What is the spin angular momentum of a single linearly polarized photon propagating in the vacuum? What is the size and shape of a linearly polarized photon? The familiar photons are mainly linearly polarized ones, but these scholars have not mentioned their size, shape, and structure.

Trouble 2-According to the formula $v=\omega r$, in all examples, the linear velocity on the rotational photon will exceed the light speed where the radius $r$ exceeds $R=\lambda / 2 \pi$.

Trouble $3-$ What is the wave-train length of a photon? In example $2,4,5$, the unreasonable conclusion is that the photon length is not equal to integral multiples of photon wavelength, or duration is not an integral multiple of the period 
$T$. It is inconsistent with the view that a photon is a complete quantum. In the theory of example 3, the author cannot determine the exact length of a photon by applying Heisenberg's uncertainty principle, so the photon length estimated from this is not the length of a single photon. Only in example 1, the photon length is equal to the wavelength, but, in reality, the radius of this photon model is infinite. After all, a single photon structure is unlikely to be the same as a hydrogen atom structure.

Moreover, only columnar (cube or cylinder) photons can link to form a continuous light-wave, but the spherical or circular ellipsoidal photons cannot.

The photon emitted by a single atom is always a linearly polarized one, namely, a single particle, while a circularly polarized photon can only exist in the light beam. The circularly polarized photon is a unit in the light beam but not a single particle. The circularly polarized light beam can only be composed of two beams of linearly polarized light with the same amplitude of electric field strength (as well as the same amplitude of magnetic field strength), perpendicular polarization planes, and phase difference of odd multiple of $\pi / 2$.

The wave function of electric field strength can understand by Fourier analysis why the wave-train length of a single photon must be equal to the wavelength. If the photon duration is not equal to an integer multiple of the period $T$, the Fourier expansion about $\omega(t-z / c)$ of the wave function (53-1) becomes an infinite series. It means that a single photon has an infinite number of frequencies. In other words, a single photon is a kind of polychromatic light but not a kind of monochromatic light. The wrong views come from pure hypothesis and imagination, while not based on theories and experiments such as Compton scattering.

Therefore, all the models of the photon above are not self-consistent.

It is well known that a single photon is the basic unit of light. Compton scattering theory is the basis for solving these problems. Finding the wavetrain length and the amplitude $E_{0}$ of the electric field strength vector are keys to find the size and shape of a single photon. Merely employing the energy formula and the spin concept is impossible to understand the electromagnetic structure.

\subsection{What Are the Methods to Determine the Size and Shape of a Single Photon?}

To solve the structural problem of a single photon can work out a scheme. Firstly, regarding the photon as a beam light and establish the wave functions such as Equations (7-1) (7-2) and (53-1) (53-2), then treat them according to quantization constraints. The size and shape of a photon are determined based on the existing knowledge of classical electrodynamics.

The first step is to assign $\hbar \omega$ to the energy of a photon and $\hbar \omega / c$ to its momentum.

The second step is to determine the wave-train length of the photon (photon 
length). Since a photon has only one frequency, the range of the photon phase can only be $|\omega(t-z / c)|=2 \pi$, that is to say, the wave-train length can only be $\mathcal{L}=\lambda$.

The third step is to determine the amplitude $E_{0}$ of electric field strength (as well as the magnetic induction strength $B_{0}$ ) using the Compton scattering formula.

The fourth step is to determine the Poynting vector using the expression of electric field strength.

The fifth step is to determine the photon's cross-sectional area using the energy formula (17-a).

The sixth step is to determine the maximum displacement of the electron in the electric field using the Compton scattering formula.

The seventh step is to determine the shape and rotation mode of the photon as a rigid body. Taking the photon as a rigid body and assigning its spin angular momentum $\hbar$, comparing the envelope with the entity part can deduce the rotational inertia formula.

Finally, synthesis of all the above parameters can construct the photon structure model.

\section{Conclusion}

A single-photon has size and shape and has an internal structure. The electromagnetic field exists only in the entity part, but not outside. A photon propagating in the vacuum has a wave-train length equal to its wavelength, namely, $\mathcal{L}=\lambda$. The shape of a linearly polarized photon is a membrane-like cuboid. The photon length in the electric field direction is $\mathcal{L}_{E}=2 \lambda / \pi$, and the photon length in the magnetic field direction is $\mathcal{L}_{M}=\alpha \lambda / 2 \pi$. Its cross-sectional area is $\sigma_{P}=\alpha \lambda^{2} / \pi^{2}$, its volume is $V_{P}=\alpha \lambda^{3} / \pi^{2}$. The magnitude of amplitude of the Poynting vector (energy flow) of the photon is $\mathcal{P}_{0}=\varepsilon_{0} c E_{0}^{2}=\hbar \omega^{4} / 4 \pi \alpha c^{2}$. The average energy density of the photon is $w=\hbar \omega^{4} / 8 \pi \alpha c^{3}$. The Compton effect probability depends on the ratio of Compton-wavelength $\lambda_{C}$ to the photon wavelength $\lambda$ when a photon with a wavelength of $\lambda$ collides with a stationary electron. The circularly polarized photon is a hollow cylinder which radius is $R=\lambda / 2 \pi$. Its electromagnetic field is confined to a membrane-like shell, while the central part is empty. The cross-sectional area of the entity part is $\sigma_{p}=\alpha \lambda^{2} / 2 \pi^{2}$, the thickness of the membrane is $\delta=\alpha \lambda / 2 \pi^{2}$.

\section{Acknowledgements}

Thank the teachers of Wuzhen High School for giving me enough time to study this topic! Thank my family for supporting my scientific research!

\section{Conflicts of Interest}

The author declares no conflicts of interest regarding the publication of this paper. 


\section{References}

[1] Compton, A.H. (1923) A Quantum Theory of the Scattering of X-Rays by Light Elements. The Physical Review, Second Series, 21, 483. https://doi.org/10.1103/PhysRev.21.483

[2] Harris, E.G. (1975) Introduction to Modern Theoretical Physics. Chapter 8, John Wiley \& Sons, Inc., 200-202.

[3] Kadin, A.M. Circular Polarization and Quantum Spin: A Unified Real-Space Picture of Photons and Electrons. https://arxiv.org/ftp/quant-ph/papers/0508/0508064.pdf.

[4] Hunter, G., Kowalski, M. and Alexandrescu, C. Einstein's Photon Concept Quantified by the Bohr Model of the Photon. http://arxiv.org/abs/quant-ph/0506231v1.

[5] Zu, D.L. (2008) The Classical Structure Model of Single Photon and Classical Point of View with regard to Wave-Particle Duality of Photon. Progress in Electromagnetics Research Letters, 1, 109-118. https://doi.org/10.2528/PIERL07111101

[6] Weidner, H. (2015) On the Size of a Photon. https://www.researchgate.net/publication/282218116 On the Size of a Photon

[7] Chen, S.N. (2016) The Nature of Photon (Structure, Size, Duality). Physical Science International Journal, 10, Article No. PSIJ.25917.

https://www.oalib.com/paper/5448073\#.YAOLA3HeogI https://doi.org/10.9734/PSIJ/2016/25917

[8] Liu, S.L. (2018) Electromagnetic Fields, Size, and Copy of a Single Photon. Arxiv: 1604.03869v3. https://arxiv.org/ftp/arxiv/papers/1604/1604.03869.pdf 